\title{
Zonificación acústica generada por decibeles no permisibles antropogénicos en la ciudad de Puno, Perú
}

\author{
Acoustic zoning generated by anthropogenic non-permissible decibels \\ in the city of Puno, Peru \\ \begin{tabular}{l|l|l} 
Recibido: febrero 11 de 2017 | Revisado: marzo 10 de 2017 | Aceptado: abril 15 de 2017
\end{tabular}
}

\author{
Grover Marín Mamani ${ }^{\mathrm{I}}$ \\ Esteban Marín PaUcara ${ }^{2}$ \\ George Argota Pérez ${ }^{3}$
}

\section{RESUMEN}

El propósito de la investigación fue determinar el comportamiento puntual y superficial del decibelio en las zonas con actividad antropogénica que generan contaminación acústica. La evaluación del decibelio se realizó por el método geoestadístico Kriging. También se utilizó el modelo Hole effect para los mapas de predicción, durante los meses de diciembre de 2014 hasta marzo de 2015. Se midieron 108 muestras de forma probabilística aleatoria, donde el $91.76 \%$ fueron puntos críticos (99) y el resto, representó una zona de confort acústico en el horario de las 07:30 a 19:30 horas. El mapa temático indicó que el factor de mayor relevancia de las seis zonas representadas fue causado por el excesivo tráfico vehicular. Se concluyó que existió contaminación acústica debido a decibeles por encima de los valores deseados.

Palabras claves: contaminación acústica, zonificación urbana, método Kriging, modelo Hole effect, PunoPerú

\section{Abstract}

The purpose of the research was to determine the punctual and surface behaviour of the decibel in zones with anthropogenic activities that generate noise pollution. The decibel is evaluated using the Kriging geostatiscal method. The Hole effect model was also used for prediction maps during the months of December 2014 and up to March 2015. 108 form samples were measured with random probability where $91.76 \%$ were critical points (99) and the rest represented an área of acoustic confort in hours from 07.30 to 19.30 hours. The thematic map indicated that the factor of greatest relevance of the six represented areas was caused by excessive vehicular traffic. It was concluded that noise pollution due to decibels above the desired values existed.

Key words: noise pollution, urban zoning, Kriging method, model Hole effect, Puno-Peru
Universidad Nacional de Juliaca (UNAJ). Puno, Perú. gmarin@unaj.edu.pe

2 Universidad Nacional del Altiplano (UNAP). Puno, Perú. esmarinpa@hotmail.com

3 Centro de Investigaciones Avanzadas y Formación Superior en Educación, Salud y Medio Ambiente "AMTAWI". Puno, Perú. george.argota@gmail.com 


\section{Introducción}

Vasilyev \& Rozenberg (2007) refieren que la contaminación acústica es uno de los problemas ambientales que preocupa a la sociedad y que el mismo puede ser descrito, a través de parámetros físicos (Chokri et al., 2017). Este fenómeno sonoro formado por vibraciones irregulares tiene como indicador el decibelio (Murphy, Edwars, Hobbs, Shepherd \& Bezombes, 2016; Shi, Su, Jin \& Liu, 2018), pues la sucesión de compresiones y enrarecimientos que provoca la onda acústica al desplazarse por el medio hace que la presión existente fluctúe en torno a su valor de equilibrio (Perelomova, 2014; Fuentes et al., 2014).

El ruido intermitente es el más constante en cualquier ambiente y espacio público, siendo el Leq, la energía acústica que contiene el ruido en determinado tiempo (Yang \& Cheng, 2016; Yousif, \& Zuri, 2017). El factor de duración en la medición estadística de la exposición al ruido se ha introducido como magnitud de nivel de presión acústica equivalente (Leq d) (Fujiwara, Meiarashi, Namikawa \& Hasebe, 2005; Makarewicz, 1984).

Por su parte, Abad, Colorado, Martin y Retana (2011) y Meyer, Layandier, Gauvreau $\&$ Benetto (2017) señalan que uno de los efectos indeseables del ruido es el estrés en el ámbito laboral y social, el cual incide negativamente en la salud humana. El MINAM (2003) refiere que los valores permisibles son $60 \mathrm{~dB}$ durante el día y $50 \mathrm{~dB}$ en la noche. En el caso de la OEFA (2011) dictaminó mediante la RCD No03-2011OEFA/CD que el valor de ruido recomendado es de $55 \mathrm{~dB}(\mathrm{~A})$, aunque este valor es superado en diversas zonas, no solo pertenecientes a la ciudad de Juliaca, provincia de San Román, sino en la propia ciudad provincial del departamento de Puno-Perú, principalmente, en el horario diurno por el tráfico vehicular (Mamani, 2013).

El propósito de la investigación fue determinar el comportamiento puntual y superficial del decibelio en zonas con actividad antropogénica que generan contaminación acústica.

\section{Materiales y Métodos}

La investigación se realizó entre los siguientes límites de coordenadas espaciales en la ciudad de Puno, Perú:

a) Norte, óvalo Yanamayo - desvío a Totorani

b) Sur, Panamericana sur, barrio Chejoña

c) Este, faldas de la cadena montańosa Señor de Huanca - las Torres

d) Oeste, bahía interior de Puno San José - Chejoña

Se realizó una observación de tipo estructurada mediante muestreo probabilístico aleatorio dentro de los límites de la investigación que comprendió una área aproximada de $13.81,854.66 \mathrm{~m}^{2}$ con perímetro de $22792.50 \mathrm{~m}$.

Se refirieron 36 estaciones de muestreo y seleccionaron 108 muestras, las cuales se georeferenciaron mediante GPS Navegador (rango de error de $2 \mathrm{~m}$ de diámetro en cada estación de monitoreo por la latitud y longitud). Para ello, se analizaron aquellos puntos críticos de contaminación acústica, entre los cuales estuvieron el tráfico vehicular, espacios públicos y sedes educativas. Se analizó el nivel de presión sonoro equivalente (Leq dB) en relación con sus coordenadas en UTM. 
El período de estudio sobre las variables estuvo determinado, a través de un análisis geoestadístico. Se utilizaron medios informáticos para la generación de mapas de predicción donde la base de datos fue elaborada a partir del monitoreo con sonómetro y procesadas en ArcGIS y se empleó el método de interpolación Kriging (coordenadas geográficas, estaciones de muestreo y nivel Leq $d B$ ).

\section{Resultados}

En la Tabla 1, se pueden observar los estadígrafos sobre los niveles de contaminación acústica (Leq $\mathrm{dB}$ ) en la ciudad de Puno.

Tabla 1

Niveles de contaminación acústica (Leq dB), ciudad de Puno

\begin{tabular}{|c|c|c|c|c|}
\hline \multicolumn{2}{|c|}{ Parámetro } & Mañana & Día & Noche \\
\hline \multicolumn{2}{|l|}{ Media } & 68.47 & 68.41 & 68.97 \\
\hline \multirow{2}{*}{$\begin{array}{l}99 \% \text { de intervalo de } \\
\text { confianza de la media }\end{array}$} & límite inferior & 65.05 & 65.64 & 64,65 \\
\hline & límite superior & 71.89 & 71.19 & 73,29 \\
\hline \multicolumn{2}{|l|}{ Media recortada al 5\% } & 69.16 & 69.00 & 69.95 \\
\hline \multicolumn{2}{|l|}{ Mediana } & 70.95 & 69.55 & 71.35 \\
\hline \multicolumn{2}{|l|}{ Varianza } & 56.80 & 37.35 & 90.67 \\
\hline \multicolumn{2}{|l|}{ Desviación estándar } & 7.54 & 6.11 & 9.522 \\
\hline \multicolumn{2}{|l|}{ Mínimo } & 46.90 & 51.1 & 40.60 \\
\hline \multicolumn{2}{|l|}{ Máximo } & 78.70 & 75.10 & 81.20 \\
\hline \multicolumn{2}{|l|}{ Rango } & 31.80 & 24.00 & 40.60 \\
\hline \multicolumn{2}{|l|}{ Rango intercuartil } & 6.20 & 5.10 & 6.70 \\
\hline \multicolumn{2}{|l|}{ Asimetría } & -1.96 & -1.87 & -2.24 \\
\hline \multicolumn{2}{|l|}{ Curtosis } & 3.86 & 3.56 & 4.96 \\
\hline
\end{tabular}

a) Si Leq $\mathrm{dB}>60 \mathrm{~dB}$ : (consideración como punto crítico, pues se sobrepasa el nivel permisible)

b) Si Leq $\mathrm{dB}<60 \mathrm{~dB}$ : (consideración como confort acústico, pues no se sobrepasa el nivel permisible)

- Leq dB mañana $=69.161 \mathrm{~dB}>60$ $\mathrm{dB}$

- Leq dB día $=69.003 \mathrm{~dB}>60 \mathrm{~dB}$

- Leq dB noche $=69.945 \mathrm{~dB}>60 \mathrm{~dB}$

La media sobre las 36 muestras tuvo una confiabilidad del $99 \%$. Los resultados indican que la hipótesis con los resultados estadísticos confirmaron que existió contaminación acústica durante el día con un rango de tiempo en el horario de las 07:30 hasta las 19:30 horas y determinaron que se superó el límite permisible.

Las zonas de concentración de ruidos superiores a 60 decibelios Leq $\mathrm{dB}$, durante la mañana indicaron que 32 muestras fueron puntos críticos y cuatro caracterizadas como de confort acústico. En el caso de Leq dB, durante el día y la noche 33 y 34 muestras fueron puntos críticos, respectivamente. De las 108 muestras seleccionadas durante el muestreo, 99 representaron puntos críticos (91.67\%). 
El análisis exploratorio con los valores sin transformación indicó que los estadígrafos referido a la media, mediana y desviación estándar no tuvieron un comportamiento normal, por lo que se efectuó una transformación logarítmica (Tabla 2).

Tabla 2

Transformación logaritmica sobre niveles de contaminación acústica (Leq dB)

\begin{tabular}{|l|c|c|c|}
\hline \multicolumn{1}{|c|}{ Variable } & Media & Mediana & $\begin{array}{c}\text { Desviación } \\
\text { estándar }\end{array}$ \\
\hline Leq dB Mańana & 4.21 & 4.26 & 0.12 \\
\hline Leq dB Día & 4.22 & 4.24 & 0.09 \\
\hline Leq dB Mañana & 4.22 & 4.26 & 0.16 \\
\hline
\end{tabular}

En la Tabla 2, el comportamiento de la variables Leq $\mathrm{dB}$ con transformación logarítmica en todos los casos indicó que se observó que la media (-) y la mediana se acercan en valores y no superan la unidad, donde la desviación estándar se encontró en un rango de 0.09 a 0.16 que es muy aceptable y los datos se normalizan para el desarrollo del método Kriging.

En la Tabla 3, puede observarse el análisis de la tendencia espacial de los datos, los cuales manifiestan tendencias direccionales que permitieron establecer correlaciones y formular modelos de comportamiento.
La tendencia de este a oeste (línea verde) fue de segundo orden (ecuación cuadrática) y mostró que los valores se comportaron con tendencia creciente hasta un pico máximo en el centro; y luego, disminuyó, gradualmente. La tendencia de datos de norte a sur (línea azul) fue de segundo orden (ecuación cuadrática) y mostró que los valores se comportaron en tendencia creciente hasta un pico máximo en el centro; y luego, disminuyó, igualmente, de forma gradual por cuanto el fenómeno sucedió para todas las muestras de las variables del Leq dB.

\section{Tabla 3}

Análisis de tendencia espacial de datos

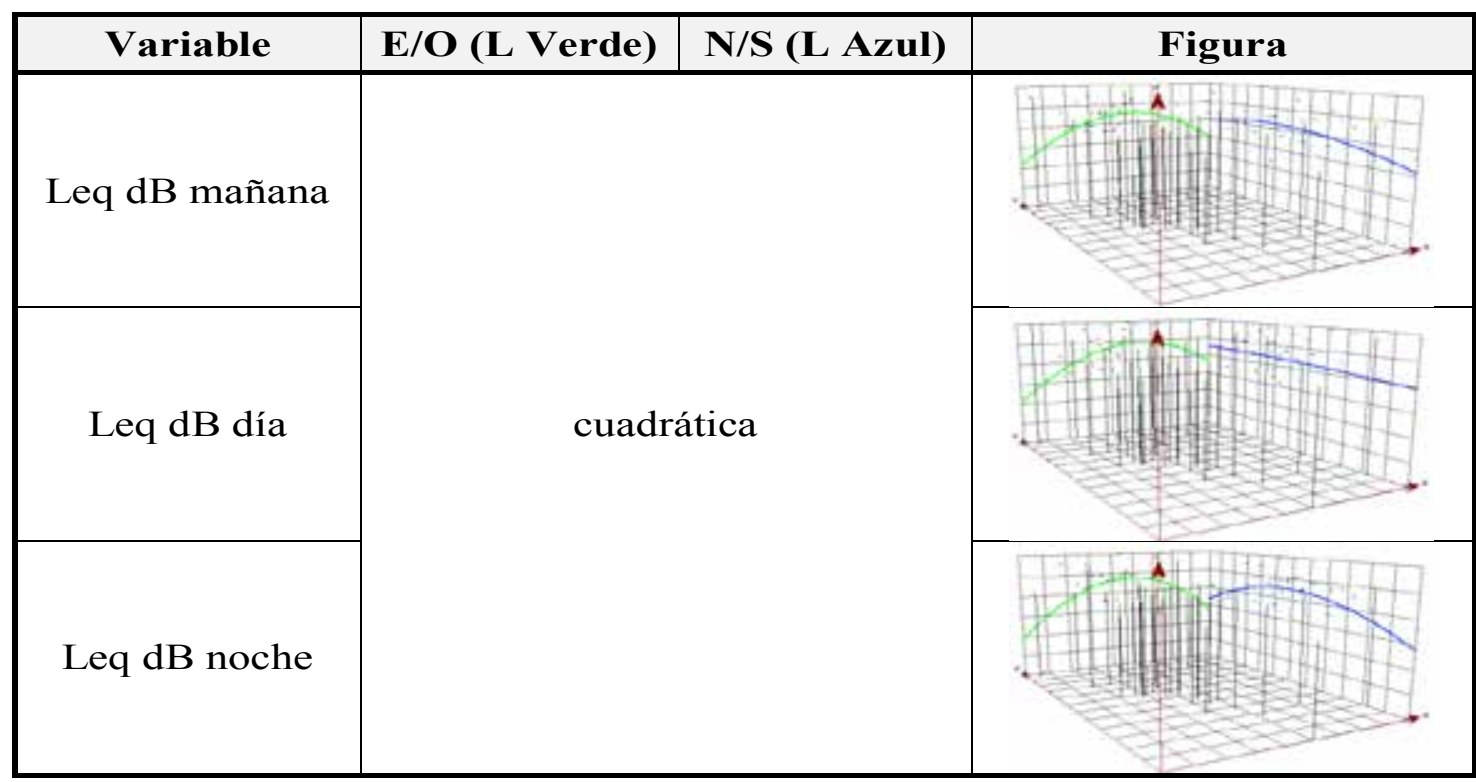


La Tabla 4 muestra la geoestadística de modelos de predicción donde el modelo Hole effect, es el que tuvo menos índices de error, por lo que se zonificó el ruido con parámetros matemáticos de este modelo. Para la selección del modelo Hole effect, se requirió efectuar un análisis estructural mediante el método Kriging, el cual arrojó mayor exactitud y nivel de confianza.

Tabla 4

Geoestadistica de modelos de predicción

\begin{tabular}{|l|c|c|c|c|}
\hline \multicolumn{1}{|c|}{ Estadística } & Esférico & Exponencial & Gauss & Hole Effect \\
\hline Mean & -0.06 & -0.60 & -0.50 & -0.60 \\
\hline Root mean square & 9.42 & 9.55 & 9.46 & 9.30 \\
\hline Mean standardized & -0.18 & -0.18 & -0.16 & -0.18 \\
\hline Root mean square standardized & 1.71 & 1.72 & 1.72 & 1.70 \\
\hline Average standard error & 4.93 & 5.14 & 4.95 & 4.90 \\
\hline Sumatoria & 15.82 & 15.63 & 15.46 & 15.12 \\
\hline
\end{tabular}

En la Tabla 5, se muestran las gráficas geoestadísticas Kriging de Leq dB, donde la predicción del modelo fue más confiable en aquellas zonas donde existen mayores estaciones de muestreo. El mayor número de errores fue para aquellas muestras que se encontraron más dispersas. Los datos tuvieron un comportamiento normal, en el centro, con una pequeña desviación en los extremos de línea.

Tabla 5

\section{Geoestadistica Kriging de Leq dB}

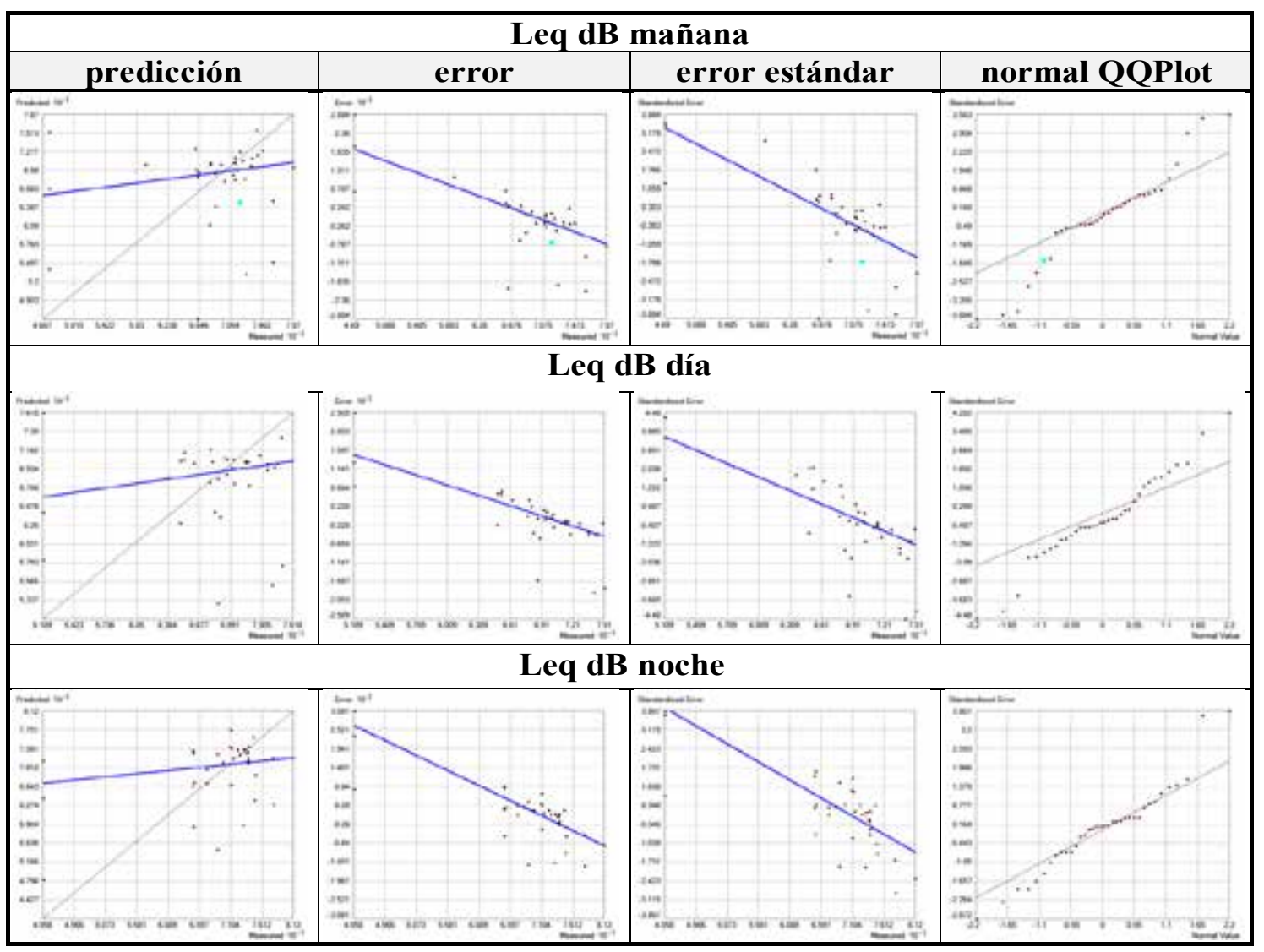


En la Tabla 6, puede observarse la zonificación Kriging de Leq dlores mínimos y máximos de $\mathrm{dB}$.

Tabla 6

Zonificación Kriging de Leq dB

\begin{tabular}{|c|c|c|c|c|c|}
\hline \multicolumn{2}{|c|}{ Leq dB mańana } & \multicolumn{2}{|c|}{ Leq dB día } & \multicolumn{2}{|c|}{ Leq dB noche } \\
\hline zonificación & error & zonificación & error & zonificación & error \\
\hline CASIFCACON & ERROR & CLASFCACONN & ERROR & CLSPCLCOON & ERROR \\
\hline$\square 34508$ & $\square 2.3 \%$ & प52508 & $\square 1.6 \%$ & П57].68 & $\square 28 \%$ \\
\hline$\square 63306$ & $\square 3 \%$ & $\square 607 \mathrm{~dB}$ & $\square 2.3 \%$ & $\square 65288$ & $\square 43 \%$ \\
\hline$\square 66.4 d 8$ & $\square .5 \%$ & $\square 663 \mathrm{~dB}$ & $\square 2.7 \%$ & $\square 685.18$ & $\square 5.1 \%$ \\
\hline$\square 67,608$ & $4.2 \%$ & $\square 70 \mathrm{~B}$ & $3.3 \%$ & $\square$ G998B & $\square 6.6 \%$ \\
\hline 口708ds & $5.3 \%$ & D250B & $4.1 \%$ & 73228 & 口 $9 \%$ \\
\hline ש79508 & च $6.9 \%$ & $\nabla 762 d 8$ & $5.3 \%$ & [10018s6 & D131\% \\
\hline
\end{tabular}

\section{Discusión}

Los valores fluctuaron entre 60 y 78 $\mathrm{dBA}$, siendo similares a los medidos en la ciudad de Juliaca (Mamani, 2013) donde el tráfico vehicular resultó de igual modo, fue la principal actividad contaminante.

Zannin, Diniz \& Barbosa (2002) indicaron que para conocer la contaminación por ruido es necesario llevar a cabo un análisis multifactorial, siendo la percepción del tiempo con las fuentes identificadas y los posibles trastornos, una de las principales variables a medir. En este caso, solo fue considerado el tráfico vehicular como una de las fuentes identificadas y que resultó, la de mayor significación. Kumar, Kumar \& Goswani (2012) encontraron, en su estudio, sobre dinámica del ruido en la ciudad de Bhadrak-India que el 46\% de los encuestados presentaron hipertensión, $48,6 \%$ habían perdido el sueño y el 52\% sufrió de irritación frecuente. Todos ellos estuvieron bajo los efectos de la contaminación acústica.

Shuokr et al., (2012) refirieron, en su estudio sobre la evaluación de la contaminación acústica del tráfico en Bukit Mertajam enMalasia y la ciudad de Erbil, que el ruido osciló entre 56.0 a 79.2 dBA. Se determinaron valores en las calles principales de 55.3 a 76.4 $\mathrm{dBA}$ mientras que, en autopistas estos se reportaron entre 62.3 a $89.2 \mathrm{dBA}$.

Resultó significativo que, durante determinadas horas, los valores estuvieron entre 66.7 a $94.8 \mathrm{dBA}$ y 64.9 a $89.1 \mathrm{dBA}$ lo que hacer suponer que debió existir algún efecto perjudicial sobre la calidad de vida humana.

El principal factor que conduce a la contaminación acústica, en la ciudad de Puno, es el tráfico vehicular ya que el $90 \%$ de las mediciones supera los 65 $\mathrm{dBA}$ y es relevante señalar que la ciudad no presenta ningún tipo de parque industrial.

Los datos medidos durante el periodo nocturno fueron menores, comparativamente, con el diurno, lo cual muestra que el ruido presenta una relación con la temperatura y al encontrarse la ciudad por encima de los $3.8 \mathrm{msnm}$ 
sus bajas temperaturas anuales, pueden amortiguar la contaminación acústica. Se concluyó que existió decibeles no permisibles en la ciudad de Puno donde las mediciones realizadas generaron una zonificación acústica.

\section{Referencias}

Abad, T.L., Colorado, A.D., Martín R.D. \& Retana, M.Ma. (2011). Ruido ambiental, seguridad $\mathrm{y}$ salud. Tecnología y Desarrollo. Revista de Ciencia, Tecnología y Medio Ambiente, 8, 1-25.

Chokri, O., Taktak, M., Zain, A., Hantati, T., Dauchez, N., Elnady, T., ... Haddar, M. (2017). Acoustic characterization of a porous absorber based on recycled sugarcane wastes. Applied Acoustics, 120, 90-97.

Fuentes, CA., Beckers, K., Pfeiffer, H., Trana, LQN., Dupont, GC., Verpoest, I \& Van Vuure, AW. (2014). Equilibrium contact angle measurements of natural fibers by an acoustic vibration technique. Colloids and Surfaces A: Physicochem. Eng. Aspects, 455, 164-173.

Fujiwara, T., Meiarashi, S., Namikawa, Y $\&$ Hasebe, M. (2005). Reduction of equivalent continuous A-weighted sound pressure levels by porous elastic road surfaces. Applied Acoustics, 66, 766-778.

Kumar, S.B., Kumar, P.S. \& Goswani, S. (2012). Dynamics of road traffic noise in Bhadrak city, India. J. Environ. Biol, 33, 1087-1092.

Makarewicz, R. (1984). Equivalent Level of Railroad Noise with and Without Barrier. Applied Acoustics, 17, 45-59.
Mamani, C.J. (2013). Evaluación de la contaminación acústica en las vias principales de la ciudad de Juliaca, Puno (Tesis de maestría). Universidad Nacional del Altiplano, Puno, Perú.

Meyer, R., Lavandier, C., Gauvreau, B \& Benetto, E. (2017). Influence of the search radius in a noise prediction software on population exposure and human health impact assessments. Applied Acoustics, 127, 63-73.

MINAM. (2003). El Reglamento de Estándares Nacionales de Calidad Ambiental para ruido (D.S. $\mathrm{N}^{\circ}$ 085-2003-PCM). Recuperado del sitio de internet del Ministerio del Ambiente del Perú: http:// w w w . m i n a m. go b. p e/ w p content/uploads/2014/02/RMN\%C2\%BA-227-2013-MINAM. pdf

Murphy, MF., Edwards, T., Hobbs, G., Shepherd, J. \& Bezombes, F. (2016). Acoustic vibration can enhance bacterial biofilm formation. Journal of Bioscience and Bioengineering, 20(20), 1-6.

OEFA. (2011). Reglamento del Procedimiento Administrativo Sancionador del Organismo de Evaluación Fiscalización Ambiental (RCD N 003-2011-OEFA/CD). Recuperado de https://www.oefa. gob.pe/?wpfb_dl=819 
Perelomova, A. (2014). Thermal selfaction effects of acoustic beam in a vibrationally relaxing gas. Appl. Math. Modelling, 38(23), 1-13. https://doi.org/10.1016/j. apm.2014.04.055

Shi, S., Su, Z., Jin, G., \& Liu, Z. (January, 2018). Vibro-acoustic modeling and analysis of a coupled acoustic system comprising a partially opened cavity coupled with a flexible plate. Mechanical Systems and Signal Processing, 98, 324-343.

Shuokr, Q.A., Lulusi, Faridah, AH., Nor, A.R., Hamidi, A.A., Amin, M. \& Umar, M. (2012). Assessment of Traffic Noise Pollution in Bukit Mertajam, Malaysia and Erbil City, Iraq. Caspian Journal of Applied Sciences Research, 1(3), 1-11.

Vasilyev, AV. \& Rozenberg, GS. (2007). Monitoring of Noise Pollution in Living Area of TogliattiCity and
Estimation of It Influence to the Health of Inhabitants. Proc. of Scientific-Practical Journal "Safety in Technosphere", 3, 9-12.

Yang, C \& Cheng, L. (2016). Prediction of noise inside an acoustic cavity of elongated shape using statistical energy analyses with spatial decay consideration. Applied Acoustics, 113, 34-38.

Yousif, A.Y. \& Zuri, S.A. (2017). Improved signal de-noising in underwater acoustic noise using Stransform: a performance evaluation and comparison with the wavelet transform. Journal of Ocean Engineering and Science, 1-29. https://doi.org/10.1016/j. joes.2017.08.003

Zannin, P.H., Diniz F.B. \& Barbosa, W.A. (2002). Environmental noise pollution in the city of Curitiba. Brazil, Appl Acoust, 63, 351-358. 


\section{GESTIÓN DE TECNOLOGÍAS}


